\title{
Enhancement of Satellite Cell Transplantation Efficiency by Leukemia Inhibitory Factor
}

\author{
N. Ito ${ }^{\mathrm{a}, \mathrm{b}}$, N. Shimizu ${ }^{\mathrm{c}}, \mathrm{H}$. Tanaka ${ }^{\mathrm{b}, \mathrm{c}}$ and S. Takeda ${ }^{\mathrm{a}, *}$ \\ ${ }^{a}$ Department of Molecular Therapy, National Institute of Neuroscience, National Center of Neurology and \\ Psychiatry, Kodaira, Japan \\ ${ }^{\mathrm{b}}$ Department of Rheumatology and Allergy, IMSUT Hospital, The Institute of Medical Science, The University \\ of Tokyo, Shirokanedai, Minato-ku, Tokyo, Japan \\ ${ }^{\mathrm{c}}$ Division of Rheumatology, Center for Antibody and Vaccine, IMSUT Hospital, The Institute of Medical Science, \\ The University of Tokyo, Shirokanedai, Minato-ku, Tokyo, Japan
}

\begin{abstract}
.
Background and Objectives: Cell transplantation is a promising therapy for several muscle diseases, including Duchenne muscular dystrophy. Satellite cells are stem cells in skeletal muscle that provide an important cell source for transplantation therapy. However, culture of satellite cells in vitro causes them to lose their undifferentiated state, associated with reduced transplantation efficiency. It is therefore necessary to develop optimal culture conditions for maintaining the undifferentiated state of satellite cells.

Methods: Primary satellite cells were cultured with or without leukemia inhibitory factor (LIF). The expression of undifferentiation and differentiation markers, and the transplantation efficiency were analyzed.

Results: LIF-treated satellite cells showed increased expression of Pax7, and enhanced transplantation efficiency in a mouse model of Duchenne muscular dystrophy.

Conclusions: Our study showed that the treatment with LIF effectively maintained the undifferentiated state of satellite cells, and enhanced their transplantation efficiency. These results will contribute to the optimization of culture conditions for cell transplantation therapy.
\end{abstract}

Keywords: Satellite cell, leukemia inhibitory factor, muscular dystrophy, cell transplantation therapy

\section{INTRODUCTION}

Duchenne muscular dystrophy (DMD) is caused by mutations in the DMD gene. DMD is the commonest form of muscular dystrophy, and is characterized by the progressive degeneration of muscle fibers and their subsequent replacement by fat or fibrotic tissue, with eventual deterioration of muscle function [1]. Although curative treatments remain to be developed, several therapies have been proposed for DMD

\footnotetext{
*Correspondence to: Shin'ichi Takeda, Department of Molecular Therapy, National Institute of Neuroscience, National Center of Neurology and Psychiatry, Kodaira 187-8502, Japan. Tel.: +81 42346 1720; Fax: +81 42346 1750; E-mail: takeda@ncnp.go.jp.
}

and other degenerative muscle diseases [2-4], including cell transplantation therapy. Satellite cells are stem cells in skeletal muscle and represent an important cell source for transplantation therapy, because their depletion leads to complete impairment of muscle regeneration $[5,6]$. Although primary satellite cells immediately after isolation shows high levels of stemness, culturing and passaging of satellite cells results in loss of their undifferentiated state, and gradual reductions in transplantation efficiency [7, 8]. However, the culture and expansion of human skeletal muscle cells in vitro is necessary to ensure the adequate cell numbers for transplantation therapy. Although growth factors, cytokines [9], and 
chemicals [10] have been used in muscle cell cultures, the optimal culture conditions required to maintain the undifferentiated state, inhibit differentiation, and enhance eventual transplantation efficiency have not yet been established.

The expression of leukemia inhibitory factor (LIF) has been reported to be increased in patients with muscular dystrophy and muscle trauma [11]. Furthermore, LIF is thought to be functionally involved in muscle regeneration, because LIF knockout mice showed impaired muscle regeneration [12], and administration of LIF ameliorated the pathology of the mouse model of DMD [13, 14]. However, although its effects on the proliferation of muscle cell lines or primary muscle cells have been studied $[12,15-18]$, the roles of LIF in the transplantation efficiency have not been analyzed. We therefore examined the effects of LIF on the undifferentiated state and transplantation efficiency in primary, freshly isolated mouse satellite cells. The results of this study will contribute to the development of optimal culture conditions for cell transplantation therapy.

\section{MATERIALS AND METHODS}

\section{Animals}

Twelve- to 16-week-old male C57BL/6 mice were purchased from Nihon CREA. Four- to 8-weekold NOD/SCID mice were purchased from Oriental Yeast. C57BL/6-green fluorescent protein (GFP)transgenic mice were kindly provided by Dr. Masaru Okabe (Osaka University, Osaka, Japan). C57BL/6$m d x$ mice were kindly provided by Dr. Toshikuni Sasaoka (National Institute for Basic Biology, Aichi, Japan). All mice were housed in the institutional animal facility. All experimental procedures were approved by the Experimental Animal Care and Use Committee at the National Center of Neurology and Psychiatry.

\section{Satellite cells preparation and culture}

Primary satellite cells were prepared from extensor digitorum longus (EDL) muscles from C57BL/6 or C57BL/6-GFP mice by dissociation with type 1 collagenase (Worthington), as described previously [19]. Briefly, isolated muscles were dissociated by incubation in $0.2 \%$ type 1 collagenase/DMEM for $80-90 \mathrm{~min}$ and unraveled by gentle pipetting. Single muscle fibers were then isolated and plated on Matrigel-coated plastic dishes. Isolated muscle fibers with primary satellite cells were cultured in DMEM (high-glucose, sodium pyruvate and GlutaMAX supplement, Thermo Fisher Scientific) supplemented with $20 \%$ fetal bovine serum, $1 \%$ chicken embryo extract (US Biological) and 1\% penicillin-streptomycin (Thermo Fisher Scientific) at $37^{\circ} \mathrm{C}$ with $5 \% \mathrm{CO}_{2}$ with or without $5 \mathrm{ng} / \mathrm{ml} \mathrm{LIF}$ (PROSPEC) for 5 days.

\section{RNA isolation and quantitative reverse transcription-polymerase chain reaction analysis}

Total RNA was isolated using TRIzol (Invitrogen) and single-strand cDNA was synthesized using a QuantiTect Reverse Transcription Kit (Qiagen). The expression levels of Pax 7, MyoD, Myogenin, and Tbp (encoding TATA-binding protein) were evaluated by quantitative reverse transcription-polymerase chain reaction (qRT-PCR) using SYBR Premix Ex Taq II (Takara) on a MyiQ single-color system (BioRad). Primer sequences for qRT-PCR were as follows: Pax7 forward: 5'-gcccacagaacctgtcactc-3', reverse: $5^{\prime}$-ggacttgaaagcttggtgctct- $3^{\prime}, M y o D$ forward: $5^{\prime}$-tgagcettgcacacctaagcc- $3^{\prime}$, reverse: $5^{\prime}$-ctccgcaagct gtggggaaa- $3^{\prime}$, Myogenin forward: $5^{\prime}$-tgtgcacatctg ttctagtctc- $3^{\prime}$, reverse: $5^{\prime}$-gctttggaaccggatagctc- $3^{\prime}, T b p$ forward: $5^{\prime}$-cagcctcagtacagcaatcaac- $3^{\prime}$, reverse: 5'taggggtcataggagtcattgg- $3^{\prime}$. The expression levels of Pax7, MyoD, and Myogenin were normalized to that of $\mathrm{Tbp}$.

\section{Satellite cells transplantation}

To induce muscle regeneration, $100 \mu \mathrm{l}$ of $10 \mu \mathrm{M}$ cardiotoxin (Sigma-Aldrich) was injected into the tibialis anterior (TA)/EDL muscles of NOD/SCID mice or $m d x$ mice 24 hours before transplantation. Thirty microliters of cell suspension containing $3 \times 10^{4}$ C57BL/6-GFP transgenic-mouse-derived satellite cells cultured with or without $5 \mathrm{ng} / \mathrm{ml}$ LIF for 5 days were injected directly into the TA/EDL muscles. After 1, 2, 4 or 14 days, the muscles were dissected, fixed in $4 \%$ paraformaldehyde for $30 \mathrm{~min}$ at $4{ }^{\circ} \mathrm{C}$, immersed in $10 \%$ sucrose/PBS followed by $20 \%$ sucrose/PBS, and then frozen in isopentane cooled with liquid nitrogen.

\section{Immunocytochemical analysis}

The obtained cells were fixed with $4 \%$ paraformaldehyde for $10 \mathrm{~min}$ at room temperature, 
washed, and treated with $0.1 \%$ Triton X-100/ phosphate-buffered saline (PBS) for $10 \mathrm{~min}$ for permeabilization. The cells were blocked with $5 \%$ goat serum (Cedarlane) in $2 \%$ bovine serum albumin (BSA)/PBS for $15 \mathrm{~min}$, and then incubated with anti-Pax7 (1:80, sc-81648, Santa Cruz), anti-MyoD (1:400, sc-32758, Santa Cruz) or anti-Myogenin antibody (1:1000, sc-12732, Santa Cruz) in $2 \%$ BSA/PBS at $4{ }^{\circ} \mathrm{C}$ overnight. The cells were then incubated with Alexa Fluor 488- or Alexa Fluor 568-labeled secondary antibodies (1:1000, Thermo Fisher Scientific) in $2 \%$ BSA/PBS. After several washings, nuclei were stained with Hoechst (DOJINDO). Immunofluorescent images were evaluated by fluorescence microscopy (Olympus).

\section{Immunohistochemical analysis}

The transplanted TA/EDL muscles were cut into $8 \mu \mathrm{m}$ transverse sections using a cryostat. The sections were fixed with cooled acetone for $10 \mathrm{~min}$ and air-dried. They were then blocked with $5 \%$ goat serum in $1 \%$ BSA/PBS for $15 \mathrm{~min}$, or M. O. M Mouse Ig Blocking reagent (VECTOR) for 1 hour followed by $5 \%$ goat serum in $1 \%$ BSA/PBS for 15 minutes. The sections were incubated with anti-GFP antibody (1:300, AB3080, Chemicon) and anti-laminin $\alpha 2$ antibody (1:200, ALX-804-190, Alexis) in 1\% BSA/PBS, or anti-GFP antibody and anti-Dystrophin antibody (1:100, Clone: Dy8/6C5, Leica) or antiGFP antibody and anti-Ki-67 antibody (1:200, Clone: B56, BD Pharmingen) in M.O.M. Diluent at $4{ }^{\circ} \mathrm{C}$ overnight. The sections were then incubated with Alexa Fluor 488- or Alexa Fluor 568-labeled secondary antibodies (1:1000, Thermo Fisher Scientific) in $1 \%$ BSA/PBS or M.O.M. Diluent. For TUNEL assay, ApopTag Red In Situ Apoptosis Detection Kit (Chemicon) was used. After several washings, nuclei were stained with DAPI (VECTOR). Immunofluorescent images were evaluated under a fluorescence microscope (BZ-9000; Keyence), and GFP-positive fibers or cells, GFP/Dystrophin doublepositive fibers, GFP/Ki-67 double-positive cells or GFP/TUNEL double-positive cells were counted.

\section{Statistical analysis}

All values were expressed as mean \pm s.e.m. Statistical differences were assessed by the Student's $t$-test. Probabilities less than $5 \%, 1 \%$ or $0.1 \%$ were considered to be statistically significant.

\section{RESULTS}

Single muscle fibers and attached primary satellite cells were cultured with or without $5 \mathrm{ng} / \mathrm{ml} \mathrm{LIF}$ for 5 days (Fig. 1A). Similar to previous report [15], the expansion of satellite cells was promoted by LIF (Fig. 1B). We first analyzed the expression of Pax7, $M y o D$ and Myogenin by qRT-PCR. We found that the expression of $\operatorname{Pax} 7$, a satellite cell marker which expression level was related to its stemness [20], was elevated by LIF (Fig. 1C). Expression of Myogenin, an essential transcription factor required for differentiation, was reduced, though the difference was not significant. These results suggest that LIF affected the maintenance of undifferentiated state in satellite cells. Expression of MyoD was slightly increased by LIF (Fig. 1C). We also found that the incidence of Pax7-negative differentiated cells were significantly decreased by LIF (Fig. 1D and E), while Myogenin-negative undifferentiated cells were increased, thus supporting a role for LIF in preventing the differentiation of satellite cells (Fig. 1D and $\mathrm{E})$.

We investigated the effects of LIF on the transplantation efficiency of satellite cells, which reflects the number of engraftable satellite cells that retained their stemness. Primary satellite cells isolated from C57BL/6-GFP mice [21] cultured with or without LIF were transplanted into cardiotoxin-injected regenerating TA/EDL muscles in NOD/SCID mice. We found that LIF-treated satellite cells formed two to three times as many GFP-positive fibers which were surrounded by laminin $\alpha 2$ as vehicle-treated satellite cells did (Fig. 2A and B). We also observed higher number of LIF-treated GFP-positive cells compared with that of vehicle-treated cells at 1,2 , or 4 days after transplantation. To elucidate the cause of this differences, we analyzed the proliferation or apoptosis of GFP-positive transplanted cells. We found that the majority of transplanted cells lost their proliferation ability immediately after transplantation (Fig. 2D and E). In addition, no difference in the number of GFP/Ki-67 double positive cells between vehicle-treated and LIF-treated satellite cells transplanted muscle was observed (Fig. 2D and E). In contrast we found that the number of GFP/TUNEL double positive cells was decreased in LIF-treated satellite cells transplanted muscle at 1 day after transplantation (Fig. 2F and G). These results suggested that treatment with LIF in vitro enhanced the transplantation efficiency of satellite cells by inhibiting their apoptosis in vivo. 
A

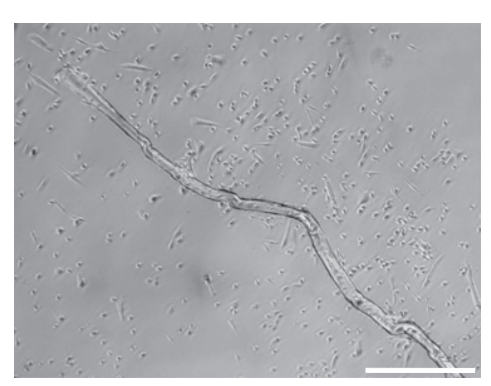

B

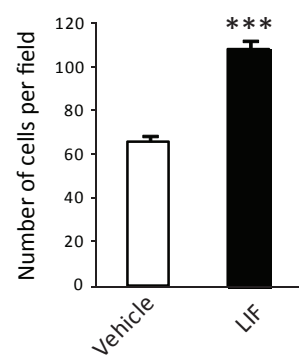

C

Pax7

MyoD

Myogenin
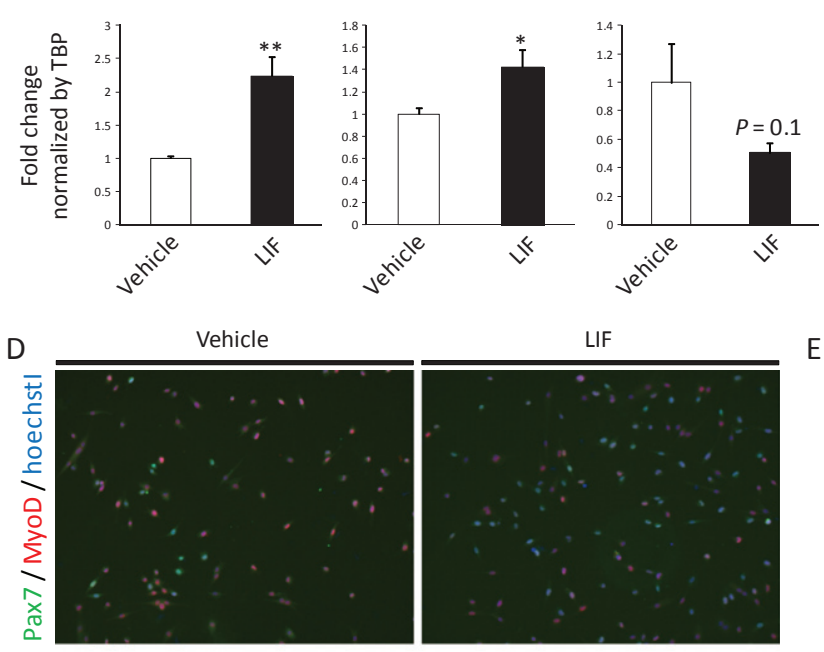

LIF

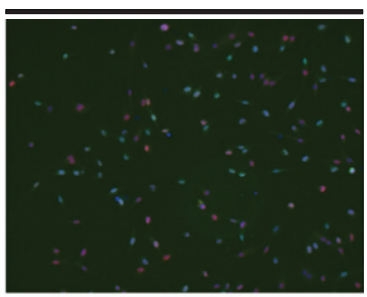

Vehicle

LIF
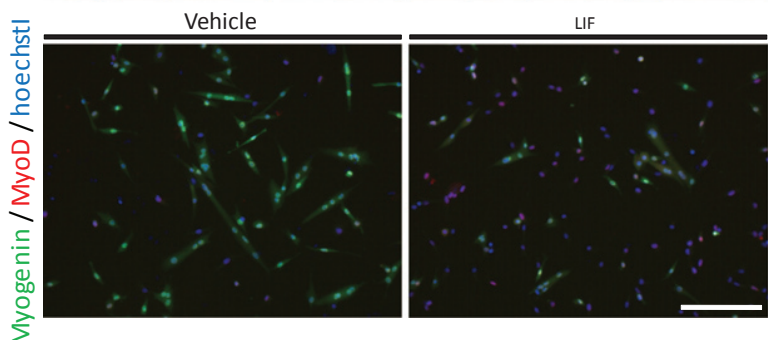

$E$
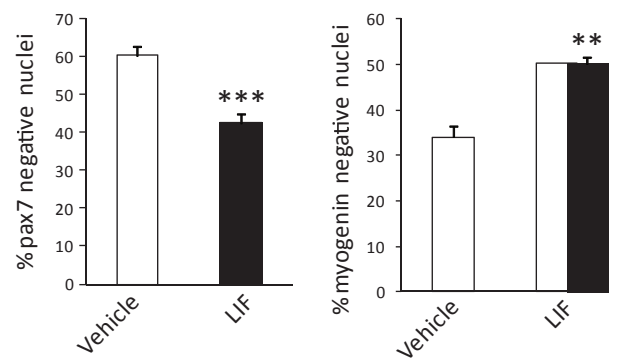

Fig. 1. LIF enhances expression of $\operatorname{Pax} 7$ in primary satellite cells. A) Representative single muscle fiber and primary satellite cells 5 days after isolation. Scale bar: $500 \mu \mathrm{m}$. B) Number of vehicle- or LIF-treated satellite cells 5 days after isolation. The number of cells per field was analyzed. $n=4$. C) Expression of Pax7, MyoD or Myogenin in vehicle- or LIF-treated satellite cells 5 days after isolation. $n=8-9$. D) Representative immunocytochemistry of Pax7 and MyoD (upper), or Myogenin and MyoD (lower) in vehicle- or LIF-treated satellite cells. Scale bar: $200 \mu \mathrm{m}$. E) Quantitative analysis of Pax7-negative (left) and Myogenin-negative nuclei (right) in vehicle- or LIF-treated satellite cells. $n=4 ;>500$ cells were analyzed per group. ${ }^{*} P<0.05,{ }^{* *} P<0.01{ }^{* * *} P<0.001$ by Student's $t$-tests. Error bars indicate standard error of mean.

Finally, we transplanted C57BL/6-GFP micederived satellite cells in $m d x$ mice, a mouse model of Duchenne muscular dystrophy. Similar to the observation in NOD/SCID mice, we observed two to three times many GFP/Dystrophin-double positive fibers in LIF-treated satellite cells-transplanted $m d x$ mice compared with those in vehicle-treated satellite cells-transplanted mice (Fig. 2H and I). Overall, these results indicated that LIF maintained the undifferentiated state, inhibited the differentiation of satellite cells in vitro, and enhanced their transplantation efficiency in vivo.

\section{DISCUSSION}

To the best of our knowledge, this study provides the first report of the effect of LIF on the transplantation efficiency of primary satellite cells. In response 
A
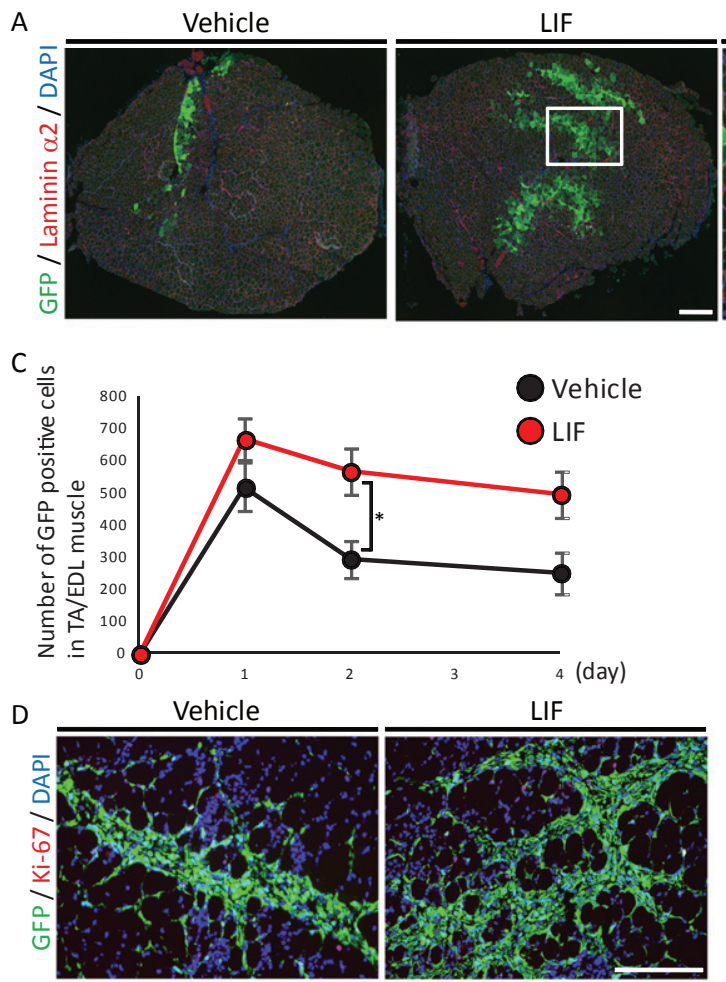

F

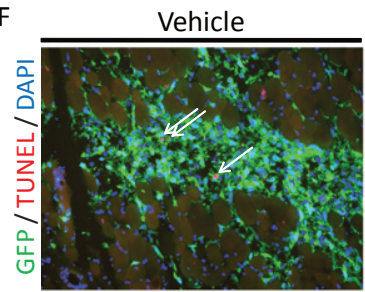

$\mathrm{H}$
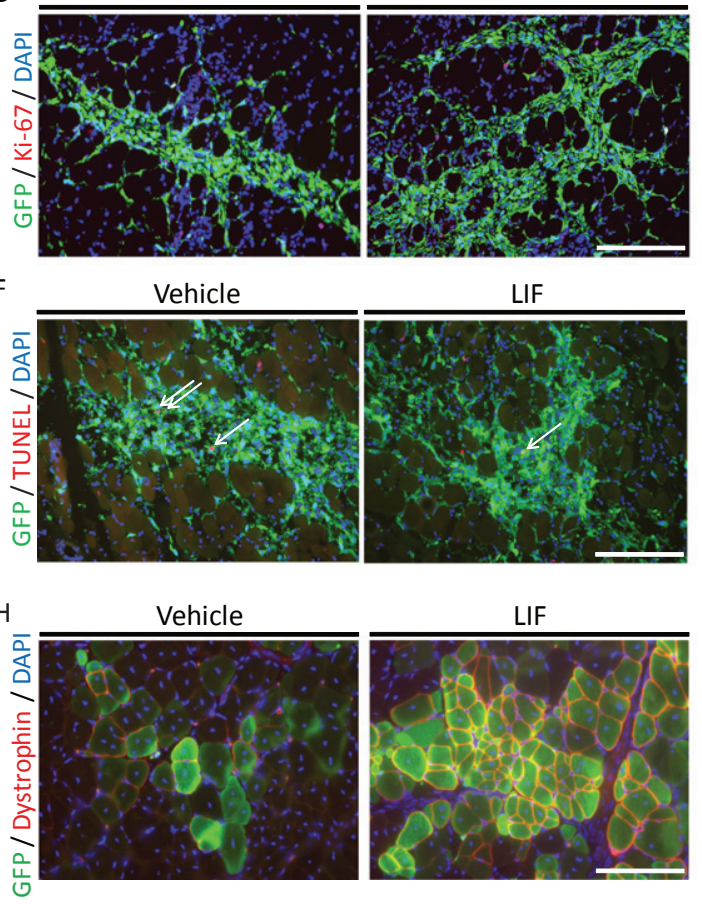

LIF (high-power field)

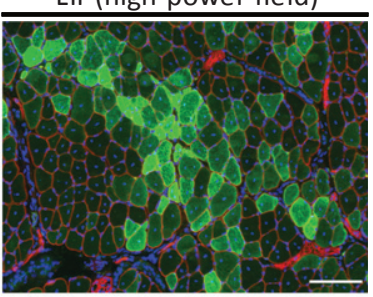

Vehicle

O LIF

LIF

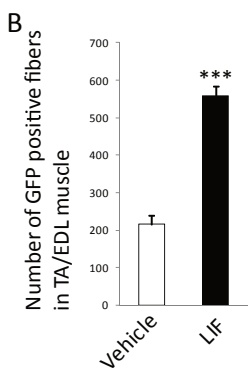

$E$

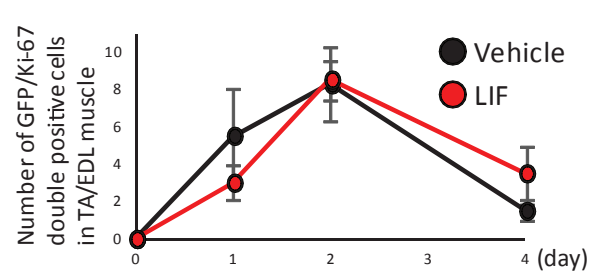

G

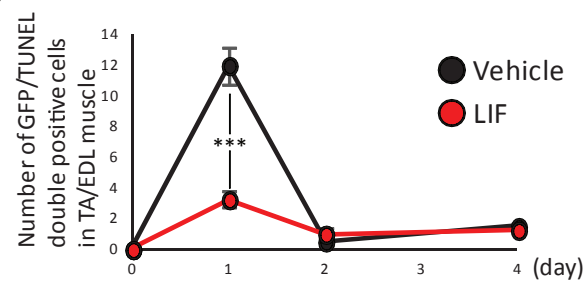

I

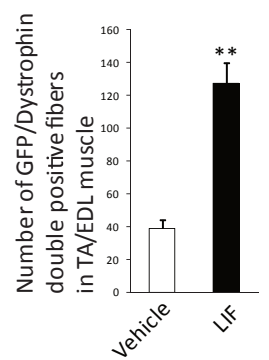

Fig. 2. LIF enhances transplantation efficiency of satellite cells. A) Representative immunohistochemistry of GFP-positive fibers in transplanted muscles at 2 weeks after transplantation. Low-power field images were shown in left and center. Scale bar: $300 \mu \mathrm{m}$. High-power field image was shown in right. Scale bar: $100 \mu \mathrm{m}$. Right image was identical to the boxed area in center image. B) Quantitative analysis for the number of GFP-positive fibers surrounded by laminin $\alpha 2$ at 2 weeks after transplantation. $n=5$. C) Quantitative analysis for the number of GFP-positive cells at 1,2 or 4 days after transplantation. D) Representative immunohistochemistry of GFP/Ki-67-double positive cells in transplanted muscles at 2 days after transplantation. Scale bar: $100 \mu \mathrm{m}$. E) Quantitative analysis for the number of GFP/Ki-67-double positive cells. $n=4$. F) Representative immunohistochemistry of GFP/TUNEL-double positive cells in transplanted cells at 1 day after transplantation. Arrows indicated GFP/TUNEL-double positive cells. Scale bar: $100 \mu \mathrm{m}$. G) Quantitative analysis for the number of GFP/TUNEL-double positive cells. $n=4$. H) Representative immunohistochemistry of GFP/Dystrophin-double positive fibers in transplanted muscles. Scale bar: $100 \mu \mathrm{m}$. I) Quantitative analysis for the number of GFP/Dystrophin-double positive fibers. $n=4$. All GFP-positive, GFP/Ki-67-double positive, GFP/TUNEL-double positive or GFP/Dystrophin-double positive cells or fibers in TA/EDL muscles were counted. ${ }^{* *} P<0.01$ ${ }^{* * *} P<0.001$ by Student's $t$-tests. Error bars indicate standard error of mean. 
to several stimuli, quiescent satellite cells are activated, proliferate, and form new muscle fibers [22]. Culturing of satellite cells lead to proliferation of their descendants, with loss of their undifferentiated state and transplantation efficiency. In addition to our results, Hashimoto et al., showed the role of LIF in the increase of round stem-like cells in vitro [23], which also support that LIF might facilitate the expansion of primary muscle cells while maintaining their undifferentiated state. However, further studies are needed to confirm the effects of LIF in long-term cultures, and in human muscle stem cells. Although we showed the effects of LIF on the enhancement of transplantation efficiency, the total number of engrafted GFP-positive fibers were approximately $3 \%$ of the total muscle fibers, and the limited distribution of transplanted satellite cells from the injected site was not improved (Fig. 2H and I), suggesting that the combination of LIF with other cytokines, such as bFGF, or transplantation with other cell population that improve migration of transplanted satellite cells would be required [24]. The use of immune deficient $m d x$ mice or immunosuppression would also enhance the survival of transplanted cells.

By analyzing the proliferation or apoptosis of transplanted cells, we found that treatment with LIF inhibited the apoptosis of transplanted cells (Fig. 2F and G), suggesting the relationship between stemness of satellite cells in vitro and their survival in vivo. However, precise mechanisms of LIF for the enhancement of transplantation efficiency remain unknown. Investigations aimed at determining the downstream targets of LIF would help to clarify the functional importance of LIF in muscle regeneration, and further its potential application in cell transplantation therapy.

\section{ACKNOWLEDGMENTS}

We thank Dr. Isao Kii and Dr. Takashi Nishiyama for valuable discussions. This work was supported by a Grant-in-Aid for Young Scientists (B) from the Japan Society for the Promotion of Science, Intramural Research Grant (28-6) for Neurological and Psychiatric Disorders of NCNP, and Research Center Network Project for Realization of Regenerative Medicine from Japan Agency for Medical Research and development (AMED).

\section{CONFLICT OF INTEREST}

The authors have no conflict of interest to report.

\section{REFERENCES}

[1] Duchenne. The Pathology of Paralysis with Muscular Degeneration (Paralysie Myosclerotique), or Paralysis with Apparent Hypertrophy. Br Med J. 1867;2(363): 541-42.

[2] Aoki Y, Yokota T, Nagata T, Nakamura A, Tanihata J, Saito T, Duguez SM, Nagaraju K, Hoffman EP, Partridge T, Takeda S. Bodywide skipping of exons 45-55 in dystrophic mdx 52 mice by systemic antisense delivery. Proc Natl Acad Sci U S A. 2012;109(34):13763-8.

[3] Shin JH, Nitahara-Kasahara Y, Hayashita-Kinoh H, Ohshima-Hosoyama S, Kinoshita K, Chiyo T, Okada $\mathrm{H}$, Okada T, Takeda S. Improvement of cardiac fibrosis in dystrophic mice by rAAV9-mediated microdystrophin transduction. Gene Ther. 2011;18(9):910-19.

[4] Xu X, Wilschut KJ, Kouklis G, Tian H, Hesse R, Garland C, Sbitany H, Hansen S, Seth R, Knott PD, Hoffman WY, Pomerantz JH. Human Satellite Cell Transplantation and Regeneration from Diverse Skeletal Muscles. Stem Cell Reports. 2015;5(3):419-34.

[5] Lepper C, Partridge TA, Fan CM. An absolute requirement for Pax 7-positive satellite cells in acute injury-induced skeletal muscle regeneration. Development. 2011;138(17): 3639-46.

[6] Relaix F, Zammit PS. Satellite cells are essential for skeletal muscle regeneration: The cell on the edge returns centre stage. Development. 2012;139(16):2845-56.

[7] Ikemoto M, Fukada S, Uezumi A, Masuda S, Miyoshi H, Yamamoto H, Wada MR, Masubuchi N, Miyagoe-Suzuki Y, Takeda S. Autologous transplantation of SM/C-2.6(+) satellite cells transduced with micro-dystrophin CS1 cDNA by lentiviral vector into mdx mice. Mol Ther. 2007;15(12): 2178-85.

[8] Quarta M, Rando TA. Mimicking the niche: Cytokines expand muscle stem cells. Cell Res. 2015;25(7):761-2.

[9] Fu X, Xiao J, Wei Y, Li S, Liu Y, Yin J, Sun K, Sun H, Wang H, Zhang Z, Zhang BT, Sheng C, Hu P. Combination of inflammation-related cytokines promotes long-term muscle stem cell expansion. Cell Res. 2015;25(6):655-73.

[10] Cosgrove BD, Gilbert PM, Porpiglia E, Mourkioti F, Lee SP, Corbel SY, Llewellyn ME, Delp SL, Blau HM. Rejuvenation of the muscle stem cell population restores strength to injured aged muscles. Nat Med. 2014;20(3):255-64.

[11] Reardon KA, Kapsa RM, Davis J, Kornberg AJ, Austin L, Choong P, Byrne E. Increased levels of leukemia inhibitory factor mRNA in muscular dystrophy and human muscle trauma. Muscle Nerve. 2000;23(6):962-66.

[12] Kurek JB, Bower JJ, Romanella M, Koentgen F, Murphy M, Austin L. The role of leukemia inhibitory factor in skeletal muscle regeneration. Muscle Nerve. 1997;20(7): 815-22.

[13] Kurek J, Bower J, Romanella M, Austin L. Leukaemia inhibitory factor treatment stimulates muscle regeneration in the mdx mouse. Neurosci Lett. 1996;212(3):167-70.

[14] Austin L, Bower JJ, Bennett TM, Lynch GS, Kapsa R, White JD, Barnard W, Gregorevic P, Byrne E. Leukemia inhibitory factor ameliorates muscle fiber degeneration in the $\mathrm{mdx}$ mouse. Muscle Nerve. 2000;23(11):1700-5.

[15] Austin L, Bower J, Kurek J, Vakakis N. Effects of leukaemia inhibitory factor and other cytokines on murine and human myoblast proliferation. J Neurol Sci. 1992;112(1-2):185191.

[16] Sun L, Ma K, Wang H, Xiao F, Gao Y, Zhang W, Wang K, Gao X, Ip N, Wu Z. JAK1-STAT1-STAT3, a key pathway 
promoting proliferation and preventing premature differentiation of myoblasts. J Cell Biol. 2007;179(1):129-38.

[17] Srikuea R, Esser KA, Pholpramool C. Leukaemia inhibitory factor is expressed in rat gastrocnemius muscle after contusion and increases proliferation of rat L6 myoblasts via c-Myc signalling. Clin Exp Pharmacol Physiol. 2011;38(8):501-9.

[18] Spangenburg EE, Booth FW. Multiple signaling pathways mediate LIF-induced skeletal muscle satellite cell proliferation. Am J Physiol Cell Physiol. 2002;283(1):C204-211.

[19] Ito N, Ruegg UT, Kudo A, Miyagoe-Suzuki Y, Takeda S. Activation of calcium signaling through Trpv1 by nNOS and peroxynitrite as a key trigger of skeletal muscle hypertrophy. Nat Med. 2013;19(1):101-6.

[20] Rocheteau P, Gayraud-Morel B, Siegl-Cachedenier I, Blasco MA, Tajbakhsh S. A subpopulation of adult skeletal muscle stem cells retains all template DNA strands after cell division. Cell. 2012;148(1-2):112-25.
[21] Okabe M, Ikawa M, Kominami K, Nakanishi T, Nishimune Y. Green mice as a source of ubiquitous green cells. FEBS Lett. 1997;407(3):313-19.

[22] Kuang S, Rudnicki MA. The emerging biology of satellite cells and their therapeutic potential. Trends Mol. Med. 2008;14(2):82-91.

[23] Hashimoto N, Murase T, Kondo S, Okuda A. InagawaOgashiwa M. Muscle reconstitution by muscle satellite cell descendants with stem cell-like properties. Development. 2004;131(21):5481-90.

[24] Motohashi N, Uezumi A, Yada E, Fukada S, Fukushima K, Imaizumi K, Miyagoe-Suzuki Y, Takeda S. Muscle CD31(-) CD45(-) Side Population Cells Promote Muscle Regeneration by Stimulating Proliferation and Migration of Myoblasts. Am J Pathol. 2008;173(3):781-91. 